\title{
Colouterine Fistula Caused by Diverticulitis of the Sigmoid Colon
}

\author{
Pyong Wha Choi \\ Department of Surgery, Inje University Ilsan Paik Hospital, Inje University College of Medicine, Goyang, Korea
}

Colouterine fistula is an extremely rare condition because the uterus is a thick, muscular organ. Here, we present a case of a colouterine fistula secondary to colonic diverticulitis. An 81-year-old woman was referred to the emergency department with abdominal pain and vaginal discharge. Computed tomography showed a myometrial abscess cavity in the uterus adherent to the thick sigmoid wall. Upon contrast injection via the cervical os for fistulography, we observed spillage of the contrast into the sigmoid colon via the uterine fundus. Inflammatory adhesion of the distal sigmoid colon to the posterior wall of the uterus was found during surgery. The colon was dissected off the uterus. Resection of the sigmoid colon, primary anastomosis, and repair of the fistula tract of the uterus were performed. The postoperative course was uneventful. This case represents an unusual type of diverticulitis complication and illustrates diagnostic procedures and surgical management for a colouterine fistula.

Keywords: Colon; Uterus; Diverticulitis; Fistula

\section{INTRODUCTION}

Colonic diverticulosis is a common disease, and its incidence increases with aging. Among patients with colonic diverticulosis, approximately $20 \%$ develop diverticulitis as a result of infection and inflammation of the diverticula [1]. The complications of diverticulitis are stricture, bleeding, perforation, and fistula formation. Among the complications of diverticulitis, fistula formation, defined as a communication between 2 epithelialized surfaces in close proximity to organs, constitutes up to $20 \%$ of the complications [1]. The most common type of fistula is a colovesical fistula followed by a colovaginal fistula arising from the sigmoid colon [2]. Because the uterus is a thick muscular organ, it provides a protective barrier against invasion of benign or malignant disease [3].

Received: December 1, 2011 - Accepted: August 9, 2012

Correspondence to: Pyong Wha Choi, M.D.

Department of Surgery, Inje University Ilsan Paik Hospital, Inje University

College of Medicine, 170 Juhwa-ro, Ilsanseo-gu, Goyang 411-706, Korea

Tel: +82-31-910-7622, Fax: +82-31-910-7319

E-mail: eacechoi@hanmail.net

(C) 2012 The Korean Society of Coloproctology

This is an open-access article distributed under the terms of the Creative Commons Attribution NonCommercial License (http://creativecommons.org/licenses/by-nc/3.0) which permits unrestricted noncommercial use, distribution, and reproduction in any medium, provided the original work is properly cited.
Therefore, a colouterine fistula is an extremely rare condition, particularly when caused by diverticulitis. Here, we present a case of a colouterine fistula caused by diverticulitis of the sigmoid colon.

\section{CASE REPORT}

An 81-year-old woman was referred to the emergency department with abdominal pain and vaginal discharge that had lasted for 4 days. Medical history included right nephrectomy for a renal stone 21 years earlier and medication for arterial hypertension 4 years earlier. The pain was localized to the lower abdomen without aggravating factors. Upon admission to the department of gynecology, the patient looked ill and had a temperature of $37.4^{\circ} \mathrm{C}$, a blood pressure of $134 / 59 \mathrm{mmHg}$, a pulse of 77 beats/min, and a respiratory rate of 18 breaths $/ \mathrm{min}$. The abdomen was slightly distended and tender over the lower abdomen, without signs of generalized peritoneal irritation. Pelvic examination showed an atrophied vagina and cervix, and a yellowish foul odorous vaginal discharge from the cervical os. Laboratory test results were within normal limits, except for a white blood cell count of $27,580 / \mu \mathrm{L}$ and a neutrophil fraction of $88.3 \%$. Culture of the vaginal discharge revealed Escherichia coli and Enterococcus avium. Endovaginal sonography showed fluid collection measuring $2.7 \times 2.4 \mathrm{~cm}$ in the endometrium, suggesting a pyometrium. Abdominopelvic com- 


\section{Coloproctology Pyong Wha choi}

puted tomography (CT) showed a myometrial abscess cavity in the uterus and diverticulosis of the sigmoid colon. Tomography also showed that the uterine had joined to the thick sigmoid wall (Fig. 1). However, no definite fistula tract was visible. A colouterine fistula caused by diverticulitis was suspected. Therefore, fistulography was performed. When contrast fluid was injected through the cervical os, spillage of the contrast fluid into the sigmoid colon

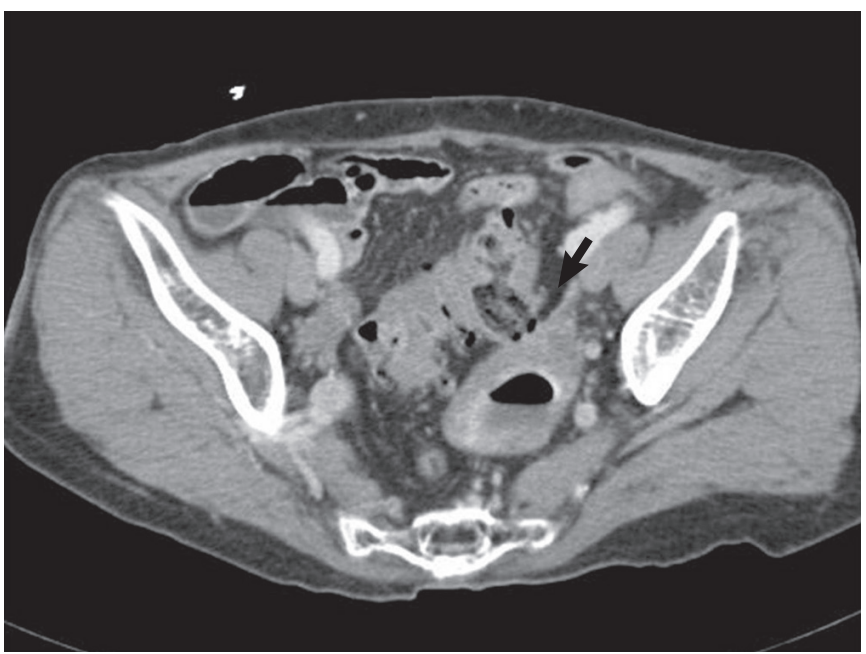

Fig. 1. Abdominopelvic computed tomography showing an air bubble in the uterine cavity with a thick sigmoid wall joined to the uterine fundus (arrow).

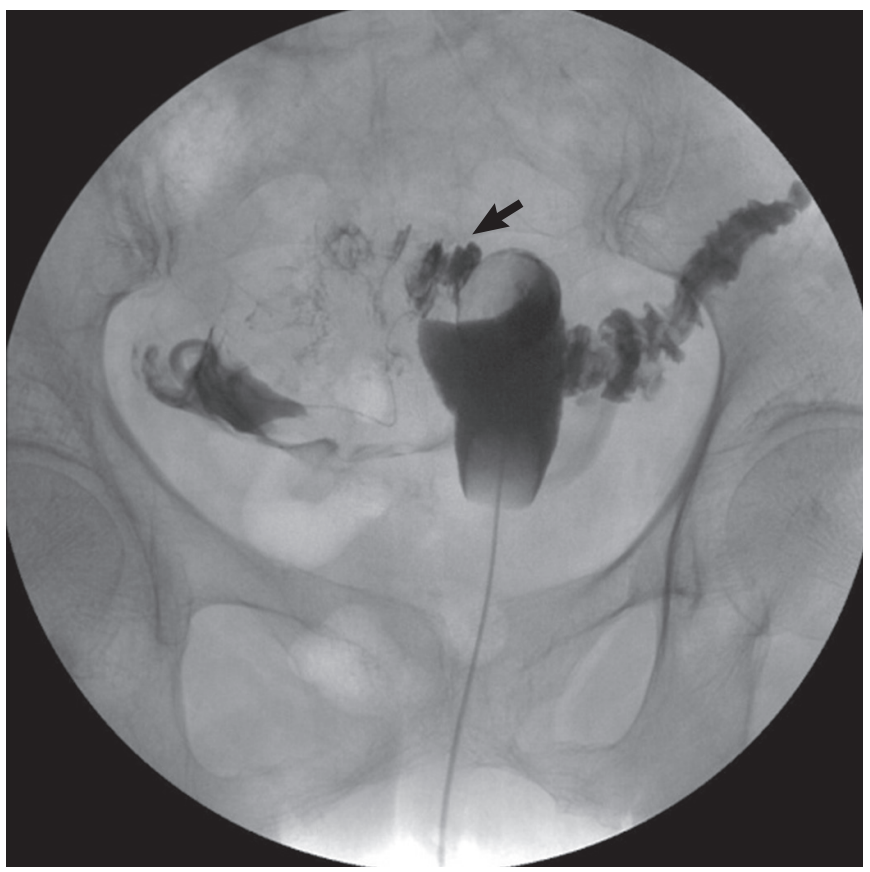

Fig. 2. Fistulography showing the spillage of the contrast medium into the sigmoid colon via the uterine fundus (arrow). via the left side of the abscess cavity of the uterine fundus was observed (Fig. 2). On colonoscopy, multiple diverticula were noted at the sigmoid colon, and a suspected lesion of the fistula opening with surrounding edema and emission of air bubbles was detected approximately $25 \mathrm{~cm}$ from the anal verge (Fig. 3). The patient was diagnosed with a colouterine fistula caused by diverticulitis and was transferred to the department of surgery for a collaborative operation.

During the operation, inflammatory adhesion of the distal sigmoid colon to the posterior wall of the uterus was found (Fig. 4). The colon was dissected off the uterus, and a fistula between the sigmoid colon and the endometrial cavity was identified. Primary

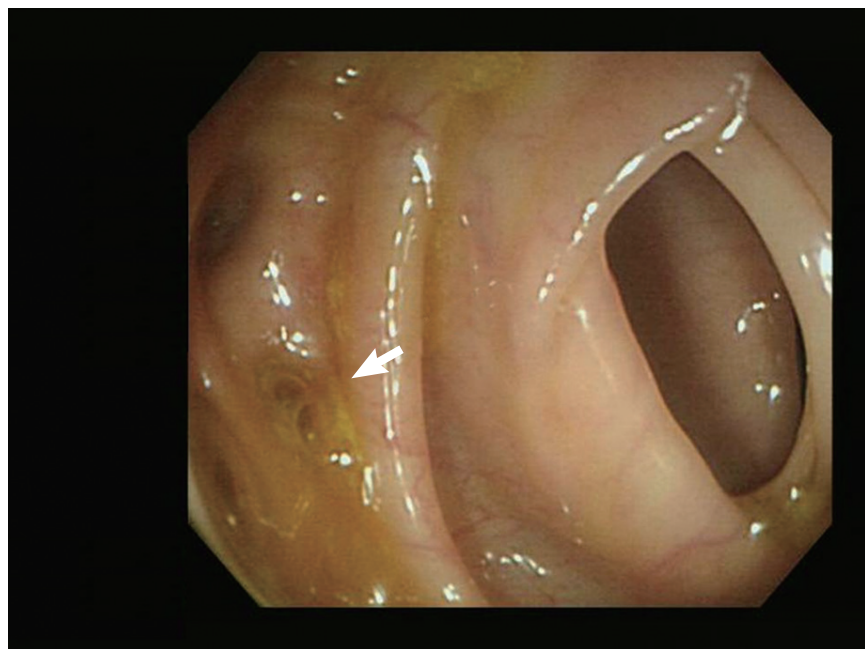

Fig. 3. Colonoscopy showing multiple diverticula and suspected lesion of the fistula opening with surrounding edema and air bubbles (arrow) approximately $25 \mathrm{~cm}$ from the anal verge.

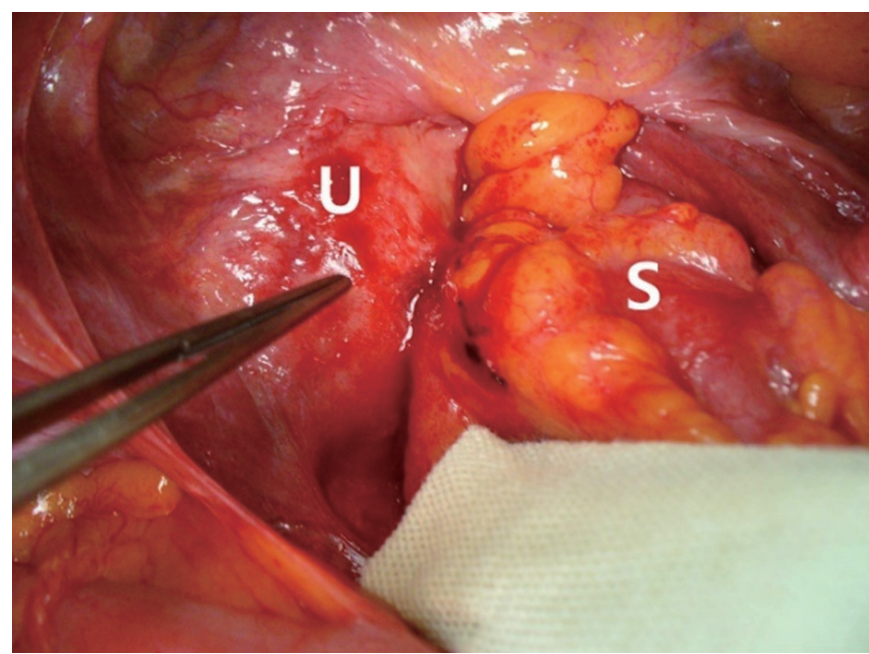

Fig. 4. Intraoperative findings. Forceps indicate inflammatory adhesion of the sigmoid colon (S) to the uterus (U). 


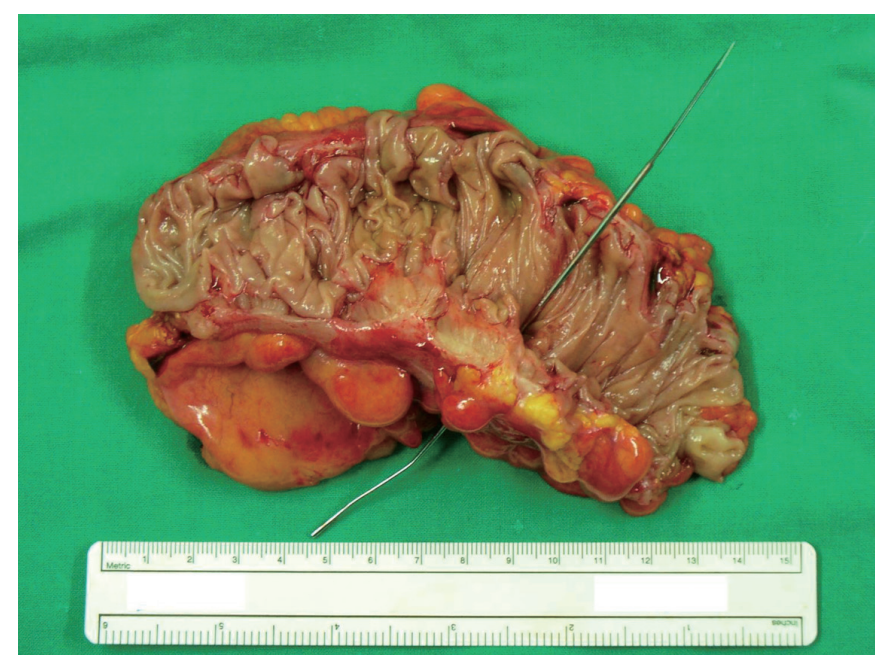

Fig. 5. Gross finding of the specimen. The excised portion of the sigmoid colon shows a fistula tract (probe) in the colonic wall.

resection of the sigmoid colon, including the affected segment, with anastomosis and repair of the fistula tract of the uterus were performed. The intrauterine purulent content was drained through the cervical os. In terms of gross appearance, the excised portion showed a fistula tract in the colonic wall (Fig. 5). The postoperative course was uneventful, and the patient was discharged on the ninth postoperative day.

\section{DISCUSSION}

Diverticulitis, defined as inflammation or infection in diverticula, is the most common complication of diverticulosis and affects approximately $20 \%$ of patients with colonic diverticula [1]. Diverticulitis of the colon may result in complications such as abscess, bleeding, obstruction, and fistula formation. Of the complications of colonic diverticulitis, fistula formation is a relatively rare complication, accounting for $20 \%$ of surgeries for complicated diverticulitis [1]. Fistula formation by colonic diverticulitis occurs when a diverticular abscess extends or ruptures into an adjacent organ such as the bladder, vagina, or small bowel $[3,4]$. A colovesicular fistula is the most common type of fistula, followed by a colovaginal fistula [2]. Because the uterus is a thick and muscular organ, it can prevent the spread of inflammation. Therefore, a colovesical fistula is more common in male than in female patients: among female patients, this condition predominantly occurs in those with a history of hysterectomy [2].

A colouterine fistula is an extremely rare type of fistula. Although the uterus provides a protective barrier against an inflammatory or malignant disease, a colouterine fistula might be caused by the spontaneous rupture of a gravid uterus, by obstetric trauma such as curettage, and uterine or sigmoid colon cancer, or by radiation therapy. Furthermore, a colouterine fistula might be caused by inflammatory processes such as periappendiceal abscess or diver- ticulitis $[5,6]$. Inflammatory adhesion of the colon and uterus can occur during an episode of diverticulitis: this results in necrosis and subsequent fistula formation, commonly resulting in linkage of the fundus of the uterus and the sigmoid colon, as we observed in this case [3].

Although clinical manifestations of a colouterine fistula vary according to causative pathology, typical symptoms include malodorous fecal or purulent vaginal discharge for days or months because the colonic lumen and the uterus are linked via a fistula tract $[3,5,6]$. Thus, diverticulitis-related symptoms such as fever and recurrent abdominal pain may be less evident or not present. In our case, the patient presented with vaginal discharge and abdominal pain at admission, but did not report a history of abdominal pain or fever. Although fecal vaginal discharge may be indicative of a colouterine or a colovaginal fistula, diagnostic modalities for a colouterine fistula have yet to be established. Vaginal or cervical cultures are necessary for determining the source of the malodorous vaginal discharge and for selecting appropriate antibiotics: these cultures may reveal the presence of gram-negative enteric organisms, as observed in this case $[5,6]$.

Many imaging modalities have been used for the diagnosis of a colouterine fistula. Generally, because patients are elderly, endovaginal ultrasonography is performed to rule out gynecologic pathologies. In our case, endovaginal sonography showed a fluid collection measuring $2.7 \times 2.4 \mathrm{~cm}$ in the endometrium, suggesting a pyometrium. However, the cause of the pyometrium could not be identified using endovaginal sonography alone. CT is increasingly being used for the evaluation of an acute abdomen and has been performed for diagnosing retroperitoneal and intraperitoneal lesions, including those involving free air, abscess, or fluid collection. In addition, CT is sensitive and specific for the diagnosis of diverticulitis and its complications [7]. In a colouterine fistula, air bubbles in the uterine cavity and colon wall joined to the uterus may be identified in CT findings. However, detection of the fistula tract or differentiation of complicated diverticulitis from colon cancer may be limited in CT findings [6]. Therefore, many imaging modalities have been suggested for the diagnosis of a colouterine fistula $[8,9]$. Although colonic lesions such as diverticulosis might be identified by using colonoscopy or a barium enema, these procedures are not helpful in the diagnosis of a colouterine fistula. However, in some cases, the colonic fistula opening may be detected, as in the present case. Kassab et al. [8] reported that magnetic resonance imaging (MRI) was a versatile, noninvasive, more sophisticated, detailed, and accurate diagnostic tool compared to CT scanning. In their report, MRI was used to identify fistulae, and T1-weighted images delineated the extension of the fistula relative to the adjacent organ and showed inflammatory changes in fat planes. The charcoal challenge test, the detection of orallyadministered charcoal flowing through the cervical os, may be used to diagnose a colouterine fistula; however, this method does not identify the fistula tract or the exact fistula site [10]. Takada et al. [9] reported that sonohysterography using a contrast medium 
could be used to visualize not only the fistula tract but also the uterine wall and the sigmoid colon. This method also prevents X-ray exposure and possible allergic response to a radio-opaque medium; thus, this diagnostic modality, instead of radiographic fistulography, may be more appropriate for a presumed colouterine fistula. In our case, CT findings suggested the diagnosis of a colouterine fistula, and fistulography was performed to obtain a definite diagnosis.

In most cases, surgical management is necessary for definite resolution of the septic focus. Various surgical approaches have been reported. The Hartman procedure with hysterectomy, a transverse colostomy followed by a hysterectomy and closure of the fistula without bowel resection, and a one-stage procedure involving en bloc resection of the uterus and sigmoid colon are several examples $[3,5,6]$. An en bloc resection, with or without diversion according to the patient's condition, is mandatory in cases of colouterine fistulae caused by malignancy. However, in benign conditions, the need for a hysterectomy has not been established. We believe that because the origin of causative pathogens in septic conditions is primarily the colon, resection of the colon alone and drainage of the purulent uterine lesion may be sufficient for definite treatment. Therefore, in cases of colouterine fistulae due to diverticulitis, separation of the uterus and the colon, followed by sigmoid colon resection and repair of the fistula tract with drainage of the uterine lesion, as performed in this case, may be an another option for surgical management. Because the prognosis for a colouterine fistula secondary to diverticulitis is excellent after surgery, selection of an appropriate surgical approach according to the patient's condition is necessary.

In conclusion, a colouterine fistula is a rare complication of diverticulitis. CT is routinely performed for diagnosis in patients with a suspected colouterine fistula. However, confirming the diagnosis by using $\mathrm{CT}$ alone might be difficult. Therefore, other noninvasive modalities such as fistulography may be necessary, and the utility of recently introduced modalities remains to be verified. Surgical approaches to a colouterine fistula include a staged operation and a one-stage operation with en bloc resection. Colon resection and repair of the fistula tract may be another option for surgical management of a colouterine fistula caused by diverticulitis.

\section{CONFLICT OF INTEREST}

No potential conflict of interest relevant to this article was reported.

\section{REFERENCES}

1. Stollman NH, Raskin JB. Diagnosis and management of diverticular disease of the colon in adults. Ad Hoc Practice Parameters Committee of the American College of Gastroenterology. Am J Gastroenterol 1999;94:3110-21.

2. Woods RJ, Lavery IC, Fazio VW, Jagelman DG, Weakley FL. Internal fistulas in diverticular disease. Dis Colon Rectum 1988;31: 591-6.

3. Chaikof EL, Cambria RP, Warshaw AL. Colouterine fistula secondary to diverticulitis. Dis Colon Rectum 1985;28:358-60.

4. Yang HY, Sun WY, Lee TG, Lee SJ. A case of colovesical fistula induced by sigmoid diverticulitis. J Korean Soc Coloproctol 2011; 27:94-8.

5. Sentilhes L, Foulatier O, Verspyck E, Roman H, Scotte M, Marpeau L. Colouterine fistula complicating diverticulitis: a case report and review of the literature. Eur J Obstet Gynecol Reprod Biol 2003;110:107-10.

6. Vilallonga R, Baena JA, Fort JM, Gonzalez O, Gemar E, Armengol Carrasco M. Colouterine fistula complicating diverticulitis in elderly women. Int J Colorectal Dis 2009;24:599-600.

7. Buckley O, Geoghegan T, O’Riordain DS, Lyburn ID, Torreggiani WC. Computed tomography in the imaging of colonic diverticulitis. Clin Radiol 2004;59:977-83.

8. Kassab A, El-Bialy G, Hashesh H, Callen P. Magnetic resonance imaging and hysteroscopy to diagnose colo-uterine fistula: a rare complication of diverticulitis. J Obstet Gynaecol Res 2008;34: 117-20.

9. Takada T, Nakagawa S, Hashimoto K, Sone K, Kugu K, Kozuma S, et al. Preoperative diagnosis of colouterine fistula secondary to diverticulitis by sonohysterography with contrast medium. Ultrasound Obstet Gynecol 2004;24:682-3.

10. Huettner PC, Finkler NJ, Welch WR. Colouterine fistula complicating diverticulitis: charcoal challenge test aids in diagnosis. Obstet Gynecol 1992;80(3 Pt 2):550-2. 